\title{
Originals
}

\section{An adequate strategy for the thermodilution technique in patients during mechanical ventilation}

\author{
J. R. C. Jansen ${ }^{1}$, J. J. Schreuder ${ }^{2}$, J. J. Settels ${ }^{3}$, J. J. Kloek ${ }^{4}$ and A. Versprille ${ }^{1}$ \\ ${ }^{1}$ Pathophysiological Laboratory, Department of Pulmonary Diseases, Erasmus University, Rotterdam, The Netherlands \\ ${ }^{2}$ Department of Anesthesiology, University of Amsterdam, Academic Medical Centre, Amsterdam, The Netherlands \\ ${ }^{3}$ TNO Biomedical Instrumentation Unit, Academic Medical Centre, Amsterdam, The Netherlands \\ ${ }^{4}$ Department of Thoracic Surgery, University of Amsterdam, Academic Medical Centre, Amsterdam, The Netherlands
}

Received: 9 April 1990; accepted: 16 May 1990

\begin{abstract}
The application of the thermodilution method in conditions associated with variations in blood flow implies a misuse of the Stewart Hamilton equation. Therefore, we studied the reliability of the thermodilution method for the estimation of mean cardiac output (CO) during mechanical ventilation in patients $(n=9)$. Variation of the injection moment in the ventilatory cycle elicited a cyclic variation of $\mathrm{CO}$ estimates. This variation was not the same for all patients neither in phase nor in amplitude. Therefore, no specific phase in the ventilatory cycle could be selected for an accurate estimation of mean $\mathrm{CO}$. Averaging $\mathrm{CO}$ estimates randomly distributed in the ventilatory cycle led to an improvement of accuracy with the square root of the number of observations. The averaging of $\mathrm{CO}$ estimates spread equally over the ventilatory cycle led to a much better result, e.g., the variation in the average of two estimates equally spread in the ventilatory cycle was similar to the variation in the average of four random estimates. We conclude that averaging of 3 or 4 estimates spread equally over the ventilatory cycle is an adequate strategy to estimate mean cardiac output in patients reliably.
\end{abstract}

Key words: Cardiac output - Mechanical ventilation Multiple injections - Thermodilution

For an accurate estimation of mean cardiac output using the thermodilution method several conditions have to be fulfilled: (i) no loss of indicator, (ii) complete mixing of indicator and blood, (iii) a constant bloodflow, and (iv) a constant baseline temperature. Under these conditions the Stewart-Hamilton equation, as incorporated in many commercial cardiac output computers, can be used. During mechanical ventilation, when bloodflow is modulated by cyclic changes in intra-thoracic pressure $[1,2]$, the Stewart-Hamilton equation is misused. In practice this may lead to a considerable scatter in random estimates of cardiac output even when the measurements are per- formed during otherwise haemodynamically stable conditions. These cardiac output values are dependent on the moment of injection of indicator in the ventilatory cycle, in pigs $[3-5]$, dogs $[6,7]$, and men $[8,9]$.

Recommendations for an accurate estimation of mean cardiac output during mechanical ventilation are contradictory. Stevens et al. [9] recommended multiple injections at the end of expiration, whereas Okamoto et al. [8] recommended paired measurements at mid-inspiration and mid-expiration. From our animal studies [3-5] we concluded that mean cardiac output could be estimated accurately by calculating the averaged value of four measurements equally spread over the ventilatory cycle. Schneider and Powner [7] confirmed this conclusion in dogs and in one patient.

The objective of the present clinical study was to evaluate the errors in the thermodilution cardiac output estimates during mechanical ventilation in patients in order to find an adequate strategy for a reliable estimation of mean cardiac output.

\section{Methods}

Nine male patients aged 55 to 67 years were studied after coronary artery bypass surgery. All suffered from multiple vessel disease, without previous myocardial infarctions, and all had stable angina pectoris with normal ventricular function. None had acute or chronic pulmonary disease. As premedication, the patients received $5 \mathrm{mg}$ of lorazepam p.o.. Anaesthesia was induced with fentanyl $(100 \mu \mathrm{g} / \mathrm{kg}$ IV), administered over 5 minutes and pancuronium bromide $(0.1 \mathrm{mg} / \mathrm{kg})$ was given to assure complete muscle relaxation. Additional small doses of fentanyl and pancuronium were used as needed. To control blood pressure after sternotomy and to facilitate rewarming after the extracorporeal circulation, sodium nitroprusside was administered $(2-4 \mu \mathrm{g} / \mathrm{kg} / \mathrm{min})$. In none of the cases were cardiac stimulants needed. The patients were ventilated with an oxygen/air mixture at a rate of $8-10$ breaths per min and an insufflation/inspiratory pause/expiratory ratio of $25 \% / 20 \% / 55 \%$. The ventilatory volume was adjusted to maintain a $\mathrm{P}_{\mathrm{a}} \mathrm{CO}_{2}$ between 32 and $42 \mathrm{mmHg}$. No positive and expiratory pressure was applied.

The instrumentation of the patients was not different from the normal clinical routine. A radial artery cannula and a $7.5 \mathrm{~F}$ Swan Ganz catheter were inserted. The Swan Ganz catheter (Edwards 93A-131 -7) was inserted via the internal jugular vein and special attention was given to the position of the thermistor in the pulmonary artery to avoid an 
extreme distal location. The thermistor was connected to a cardiac output computer (Edwards COM 1).

\section{Measurements and estimation of cardiac output}

Electrocardiogram, radial arterial pressure, pulmonary arterial pressure, central venous pressure, tracheal pressure, ventilatory flow, and body temperature were monitored on a chart recorder (Gould ES 1000) to check the stability of the patients during a series of 12 measurements. Patients with a change in one of the pressures of more than $5 \%$ over the period of a series were excluded from the study.

Injection of $5 \mathrm{ml}$ glucose solution $(5 \%)$ at room temperature was automatically performed by a phase controller and a pneumatically driven syringe, after a manual start of the cardiac output computer. The injectate was delivered through the Swan Ganz catheter, within $1 \mathrm{~s}$. After 12 seconds the syringe was automatically refilled. The moment of injection was dependent on a start signal given by the operator and the moment in the ventilatory cycle set on the phase controller. The moment in the ventilatory cycle was derived from the Siemens servo ventilator (900 B). This ventilator delivers 100 impulses during each ventilatory cycle giving a subdivision in percentages. These impulses were used to feed a counter in the phase control unit. The counter was reset at the start of each insufflation.

\section{Experimental protocol}

A series of twelve thermodilution measurements was carried out during haemodynamically stable conditions. A cardiac output measurement was repeated if the COM 1 computer showed an alert signal and the tracing of the dilution signal showed an abnormal curve. At least five ventilatory cycles were inserted in between two measurements, so each series was performed in approximately $9-10$ minutes. The injections were done successively at the phases $0 \%, 25 \%, 50 \%, 75 \%, 8 \%, 33 \%$, $58 \%, 83 \%, 17 \%, 42 \%, 67 \%$, and $92 \%$ of the ventilatory cycle, where phase zero was chosen at the start of insufflation. The mean of all twelve cardiac output estimates was accepted to be the real mean cardiac output. Each measurement was expressed as a percentage of this mean value.

\section{Averaging of estimates}

Two types of selection procedures were used: a systematic selection and a random selection of single estimates (Fig. 1), as described in detail before [4] and briefly summarized here.

\section{Systematic selection}

A two-point-average was obtained by the average of two points half a ventilatory cycle apart. There were 6 such two-point-averages available from a series of 12 single estimates, i.e., $0 \%+50 \%, 8 \%+58 \%$, etc. up to $42 \%+92 \%$. Four three-point-averages were calculated similarly, i.e., $0 \%+33 \%+67 \%, 8 \%+42 \%+75 \%, 17 \%+50 \%+83 \%$, and $25 \%+58 \%$ $+92 \%$. The three four-point-averages per series were obtained from the phases $0 \%+25 \%+50 \%+75 \%, 8 \%+33 \%+58 \%+83 \%$, and $17 \%+$ $42 \%+67 \%+92 \%$, respectively.

\section{Random selection}

As in the systematic procedures again two- to four-point-averages were calculated. For example a four-point-average was obtained by taking four random single estimates, each from the full series of twelve. Thus, it was possible to select, by chance, the same value four times. This random selection was not completely analogous to four injections at the same phase in the ventilatory cycle, because the estimates would then have been mutually different due to instability of the patients and measurement errors.

\section{Statistical analysis}

$p$-levels for differences between measurements within the same patient were calculated according to a paired Student's $t$-test for small samples. Significance was determined at $p<0.05$.

\section{Results}

In all nine patients the haemodynamic variables (Table 1) were stable for the series of 12 observations. Mean values \pm SD were for radial artery pressure $89 \pm 5 \mathrm{mmHg}$, pulmonary arterial pressure $19 \pm 4 \mathrm{mmHg}$, and central venous pressure $9 \pm 1 \mathrm{mmHg}$.

After sorting the series of 12 measurements with respect to the moments of injection in the ventilatory cycle a cyclic pattern of modulation of the estimates appeared. Figure 2 shows the results of three selected series of $12 \mathrm{CO}$ estimates for 3 patients. All these patterns of modulation have the same periodicity as the ventilation, but are shifted in phase $(\Phi)$ and have different amplitudes of variation. In this figure we have indicated the point where the curves crossed the $100 \%$ line in the negative direction. This point has been expressed as a percentage of the whole cycle, starting with insufflation. In agreement with this figure for three patients we observed marked differences in the amplitude and the phase of the pattern of modulation for all patients.

The variance in the phase $(\Phi)$ for the nine patients is given in Table 2, together with the mimimum $\mathrm{CO}_{\min }$ and maximum $\left(\mathrm{CO}_{\max }\right)$ values of the single estimates in percentages of the mean cardiac output. The maximum dif-

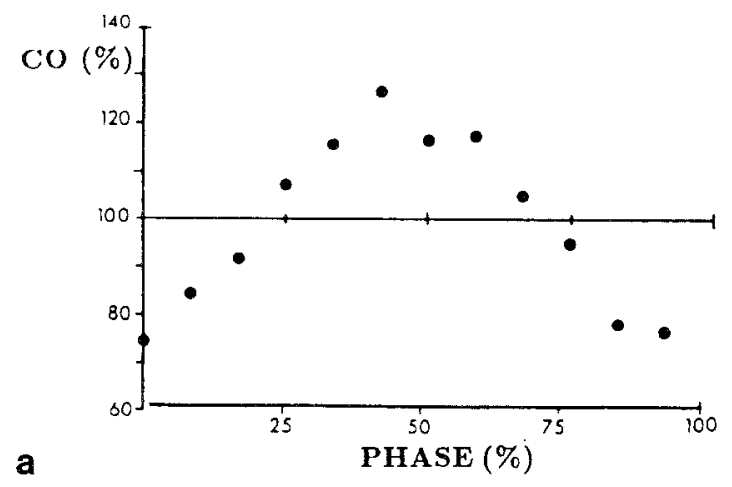

Systematic selection

Random selection

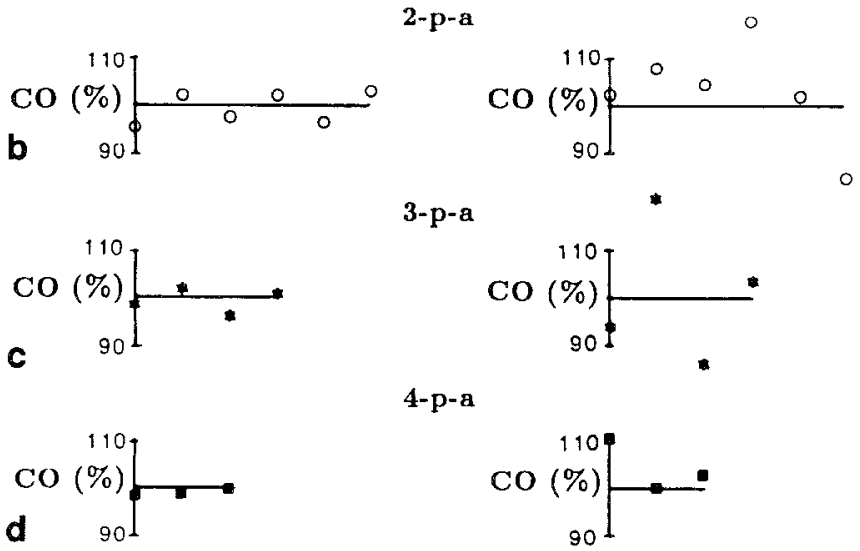

Fig. 1. An example of the averaging techniques. Values are given in $\%$ of the mean of a series of all 12 single estimates. a 12 single estimates of a patient plotted against the moment of injection in the ventilatory cycle. Phase $100 \%$ is the same as phase $0 \%$ which coincide with the start of insuffation. b 6 two-point-averaged (2-p-a) values consecutively plotted on the horizontal axis, c 4 three-point-averaged (3-p-a) values. d again, 3 four-point-averaged (4-p-a) values. For further explanation see text 
Table 1. Data of the nine patients in the study. CO, mean to twelve cardiac output estimates; $P_{r a}$, arterial pressure measured in the radial artery; $\mathrm{P}_{\mathrm{pa}}$, pulmonary artery pressure; $\mathrm{P}_{\mathrm{cv}}$, central venous pressure; $\mathrm{SD}$, standard deviation of the mean

\begin{tabular}{lllllll}
\hline Patient & $\begin{array}{l}\mathrm{CO} \\
\mathrm{l} / \mathrm{min}\end{array}$ & $\begin{array}{l}\mathrm{P}_{\mathrm{ra}} \\
\mathrm{mmHg}\end{array}$ & $\begin{array}{l}\mathrm{P}_{\mathrm{pa}} \\
\mathrm{mmHg}\end{array}$ & $\begin{array}{l}\mathrm{P}_{\mathrm{cv}} \\
\mathrm{mmHg}\end{array}$ & $\begin{array}{l}\text { Weight } \\
\mathrm{kg}\end{array}$ & $\begin{array}{l}\text { Age } \\
\text { years }\end{array}$ \\
\hline 1 & 3.35 & 90 & 28 & 12 & 78 & 55 \\
2 & 5.27 & 97 & 20 & 11 & 90 & 61 \\
3 & 5.78 & 94 & 19 & 10 & 82 & 67 \\
4 & 4.42 & 93 & 20 & 9 & 75 & 56 \\
5 & 6.15 & 79 & 16 & 8 & 74 & 59 \\
6 & 3.83 & 90 & 19 & 10 & 83 & 62 \\
7 & 3.54 & 87 & 22 & 8 & 68 & 64 \\
8 & 3.97 & 84 & 15 & 9 & 94 & 55 \\
9 & 4.14 & 88 & 15 & 8 & 78 & 57 \\
Mean & 4.49 & 89 & 19 & 9 & 80 & 60 \\
SD & 1.00 & 5 & 4 & 1 & 8 & 4 \\
\hline
\end{tabular}

ference between the largest and the smallest values of the estimates in a series of 12 measurements in a patient was $58 \%$. The mimimum difference was $29 \%$.

The results of the averaging procedures for all series are presented in Table 3. In the systematic procedures all mean values were, by definition, $100 \%$, because all measurements are always involved. In the randomly selected population the mean was slightly different from $100 \%$, because some data points were selected more than once and others not at all.

Taking a random set of cardiac output estimates gave approximately the same mean and variance as the total original set of estimates with an SD of approximately $13 \%$. The standard deviation decreased close to a factor $\sqrt{2}$ for the randomly selected two-point averages and with a factor 2 for the systematically selected two-point averages. The largest improvement in accuracy of the estimation of cardiac output can be seen in the systematically selected estimates for the two- and three-point averages. The further improvement in accuracy when using 4 estimates was smaller. Averaging three or four estimates equally spread in the ventilatory cycle gave almost $100 \%$

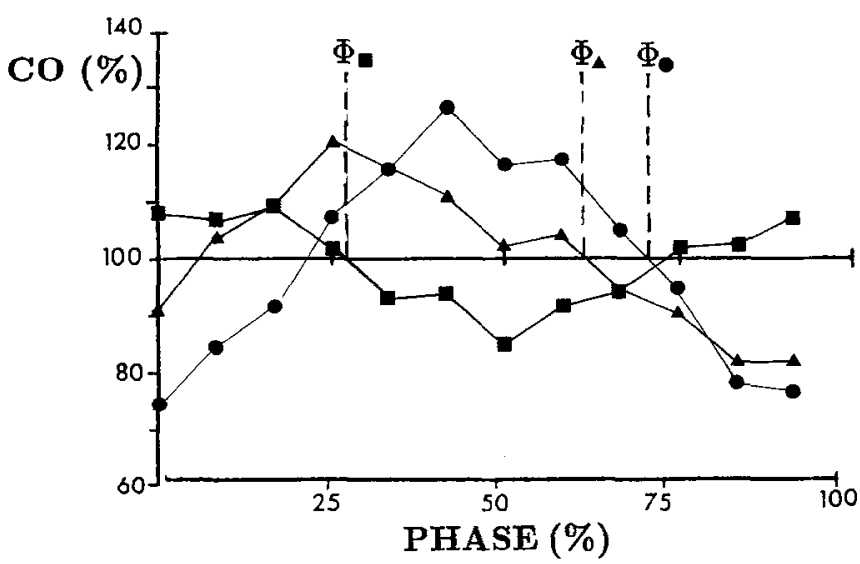

Fig. 2. Three individual series of 12 cardiac output (CO) measurements in three patients, plotted against the moment of injection as a percentage phase of the ventilatory cycle. $100 \%$ is the mean of each series of 12 estimates. $\Phi_{\Lambda}, \Phi_{\square}$ and $\Phi_{\bullet}$ are the phases at which the $100 \%$ value is crossed in negative direction
Table 2. Phase and amplitude of the modulation in the nine patients. $\Phi$, phase at which the $\mathrm{CO}$ variation in the ventilatory cycle crossed the $100 \%$ in negative direction, see also Fig. 2. $\mathrm{CO}_{\min , \max }$, minimal and maximal values for a series of 12 estimates; SD, standard deviation of the mean

\begin{tabular}{llll}
\hline Patient & $\Phi(\%)$ & $\mathrm{CO}_{\text {min }}(\%)$ & $\mathrm{CO}_{\max }(\%)$ \\
\hline 1 & 33 & 83 & 113 \\
2 & 33 & 82 & 130 \\
3 & 27 & 75 & 128 \\
4 & 27 & 83 & 114 \\
5 & 50 & 85 & 114 \\
6 & 62 & 82 & 121 \\
7 & 71 & 80 & 112 \\
8 & 70 & 71 & 129 \\
9 & 71 & 76 & 127 \\
Mean & 49 & 80 & 121 \\
SD & 20 & 5 & 8 \\
\hline
\end{tabular}

of all values within the $10 \%$ confidence limits and approximately $90 \%$ within the $5 \%$ confidence limits.

\section{Discussion}

Although, under the circumstances of mechanical ventilation, the Stewart-Hamilton equation is not valid, cardiac output computers based on this formula are applied clinically. We studied the errors from this type of application to find a solution for this theoretical misuse.

\section{Cyclic variation in cardiac output}

Our study in patients showed the presence of a cyclic modulation in cardiac output estimates related to ventilation (Figs. 1 and 2). This result is in agreement with the data found in animals $[3,4,6,7]$ and humans $[8,9]$. On the cyclic modulation a random variation was present. This random variation may be caused by (i) haemodynamic instability of the patients [11], (ii) slow and rapid baseline temperature fluctuations during the measurement, and (iii) errors in the cardiac output device. Repeated measurements at a chosen moment in the ventilatory cycle will decrease the random errors for a specific patient. But the choice of one moment for cardiac output estimation will give different values for different pa-

Table 3. Averaging techiques. 1-s-e, single estimates; 2-p-a, twopoint-averages; 3-p-a, three-point-averages; 4-p-a, four-point-averages. $\pm 10 \%, \pm 5 \%$, percentage of the total number of measurements within $10 \%$ and $5 \%$ accuracy respectively; $n$, number of values; SD, standard deviation of the mean

\begin{tabular}{|c|c|c|c|c|c|c|c|c|c|}
\hline & \multicolumn{4}{|c|}{ Systematic } & \multicolumn{5}{|c|}{ Random } \\
\hline & $\begin{array}{l}\text { Mean } \\
(\%))\end{array}$ & $\begin{array}{l}\text { SD } \\
(\%)\end{array}$ & $\begin{array}{l}\% \text { of } \\
\text { data } \\
\text { within } \\
\pm 10 \%\end{array}$ & $\begin{array}{l}\% \text { of } \\
\text { data } \\
\text { within } \\
\pm 5 \%\end{array}$ & $\begin{array}{l}\text { Mean } \\
(\%))\end{array}$ & $\begin{array}{l}\mathrm{SD} \\
(\%)\end{array}$ & $\begin{array}{l}0 \% \text { of } \\
\text { data } \\
\text { within } \\
\pm 10 \%\end{array}$ & $\begin{array}{l}\% \text { of } \\
\text { data } \\
\text { within } \\
\pm 5 \%\end{array}$ & $\mathrm{n}$ \\
\hline $1-\mathrm{s}-\mathrm{e}$ & 100.0 & 13.0 & 58 & 34 & 101.8 & 13.9 & 57 & 28 & 108 \\
\hline $2-p-a$ & 100.0 & 6.1 & 89 & 69 & 102.8 & 9.7 & 67 & 44 & 54 \\
\hline $3-p-a$ & 100.0 & 3.2 & 100 & 89 & 101.4 & 7.2 & 83 & 53 & 36 \\
\hline 4-p-a & 100.0 & 3.1 & 100 & 89 & 100.3 & 5.7 & 93 & 59 & 27 \\
\hline
\end{tabular}


tients, because of the inter-individual differences in modulation pattern of the cardiac output estimates (Fig. 2 and Table 2).

\section{Monitoring of changes in cardiac output}

It is believed that relative changes in $\mathrm{CO}$ can be followed if injections are made at a fixed moment in the ventilatory cycle $[9,10]$. This approach needs a constant phase relationship in $\mathrm{CO}$ variations versus ventilatory cycle. It was demonstrated that the phase relationship between the $\mathrm{CO}$ estimates and the ventilatory cycle is changed, when either ventilatory pattern, frequency, end expiratory pressure or blood volume conditions are changed $[3,4]$. In this study we observed different patterns and phase relationships of the $\mathrm{CO}$ estimates in the ventilatory cycle for different patients, which were presumably due to differences in haemodynamic conditions.

\section{Averaging of estimates}

To determine cardiac output clinically a general accepted method is the averaging of multiple random measurements. From animal studies [3, 4, 7], where we compared the thermodilution method and the Fick method, we know that the mean of 12 estimates equally spread over the ventilatory cycle represents an accurate estimate of the mean cardiac output. We assumed that the mean of twelve such measurements in our patients represented mean cardiac output.

Averaging $\mathrm{CO}$ estimates of randomly distributed injections led to an improvement in accuracy with the square root of the increase in number of oberservations. Thus, the average of two random estimates improves accuracy with a factor 1.4 compared with the single average of random estimates. Actually, the random selection was restricted to measurements at twelve different, but equally spread, moments in the ventilatory cycle. We have good evidence to regard these twelve estimates as a representative substitute for estimates at all moments in the ventilatory cycle. Firstly, the improvement in the accuracy with a factor $\sqrt{2}$ when doubling the number of estimates for averaging indicates a normal distribution of all twelve estimates. Secondly, the improvement in the estimation of cardiac output by an increasing number of single estimates randomly selected from the 12 estimates is similar to that found in the same procedures selected from 50 estimates in animal studies [4].

A better result was obtained from the averaging of systematically selected $\mathrm{CO}$ estimates. The average of two estimates with a phase difference of half a ventilatory cycle proved as accurate as the average of four random estimates. Because of the superior properties of the systematic averaging technique as shown in Table 3 we rejected the recommendation of Stetz et al. [10] to average three randomly performed observations. Okamoto et al. [8] confirmed our rejection $[3,4]$ of cardiac output measurements only made at the end of expiration, and recommended the estimation of mean cardiac output by calculating the average of two measurements, one performed half way during insufflation and one half way during expiration. These points in the ventilatory cycle could be synchronized "by hand" for injections by listening to noise of the ventilator and by monitoring either intratracheal pressure or chest wall movements. We would only follow the recommendation of Okamoto et al. when these points in the ventilatory cycle have a difference of half a cycle.

It has been argued that measurements equally spread over the ventilatory cycle are a technically demanding exercise [9]. However, our instrumentation was relatively simple, and can be easily incorporated in new cardiac output computers.

Based on the presented clinical study and on our results from animal experiments [3-5] we concluded that mean cardiac output can be best estimated by averaging 3 or 4 measurements initiated at 3 or 4 equally spaced intervals over the ventilatory cycle. This will reduce both random noise and systematic deviations to an acceptable level of accuracy for follow-up in one patient and mutual comparison of results between different patients.

\section{References}

1. Hoffman JIE, Guz A, Charlier AA, Wilcken DEL (1965) Stroke volume in conscious dogs: effect of respiration, posture and vascular occlusion. J Appl Physiol 20:865-877

2. Morgan BC, Martin WE, Hornbein TF, Crawford EW, Fronek A (1966) Hemodynamic effects of intermittent positive pressure ventilation with and without an end-expiratory pause. Anesthesiology $27: 584-590$

3. Jansen JRC, Schreuder JJ, Bogaard JM, v Rooyen W, Versprille A (1981) The thermodilution technique for the measurement of cardiac output during artificial ventilation. J Appl Physiol 51:584-591

4. Jansen JRC, Versprille A (1986) Improvement of cardiac output estimation by the thermodilution method during mechanical ventilation. Intensive Care Med 12:71 - 79

5. Jansen JRC, Bogaard JM, Versprille A (1987) Extrapolation of thermodilution curves obtained during a pause in artificial ventilation. J Appl Physiol 63:1551-1557

6. Armengol J, Man GCW, Balsys AJ (1981) Effects of the respiratory cycle on cardiac output measurements: reproducibility of data enhanced by timing the thermodilution injections in dogs. Crit Care Med 9:852-854

7. Snyder JV, Powner DJ (1982) Effects of mechanical ventilation on the measurement of cardiac output by thermodilution. Crit Care Med 10:677-682

8. Okamoto K, Komatsu T, Kumar V, Sanchala V, Kabul K, Bhalodia R, Shibutani K (1986) Effects of intermittent positive-pressure ventilation on cardiac output measurements by thermodilution. Crit Care Med 14:977-980

9. Stevens JH, Raffin TA, Mihm FG, Rosenthal MH, Stetz CW (1985) Thermodilution cardiac output measurement. Effect of the respiratory cycle on its reproducibility. JAMA 253:2240-2242

10. Stetz CW, Miller RG, Kelly GE (1982) Reliability of the thermodilution method in the determination of cardiac output in clinical practice. Am Rev Respir Dis 125:1001-1004

11. Wessel HU, Paul MH, James GW, Grahn AR (1971) Limitations of thermal dilution curves for cardiac output determinations. J Appl Physiol 30:643-652

Dr. J. R. C. Jansen

Department of Pulmonary Diseases

Erasmus University

Room Ee2251

P.O. Box 1738

NL-3000 DR Rotterdam

The Netherlands 